\title{
Introduction of a medical utilisation manager and the use of exception reporting to enable mandatory CMT clinic attendance. An educational chief registrar quality improvement project
}

\author{
Authors: Lisa Waters* and Matthew Foster
}

\begin{abstract}
Background
Regular attendance at outpatient clinics is an important educational experience for core medical trainees (CMTs) and is a mandatory requirement for the Annual Review of Competence Progression (ARCP). CMTS are required to attend 40 outpatient clinics over the 2-year period. During the 2017/18 academic year at Warrington Hospital there was considerable dissatisfaction among the CMTs contributing to a red RAG rating in the annual GMC training survey. The medical rota did not specifically allocate time for clinics and trainees were often unable to leave the ward to attend clinic due to staffing shortages. These issues were highlighted to the trust postgraduate education team. There was no formal data collection in place to ascertain which clinics were available and which trainees had attended.
\end{abstract}

\section{Materials and methods}

Changes were implemented. A medical utilisation manager (MUM) was employed and tasked with devising a rota for each CMT at the start of their placement. Key stakeholders were engaged including medical consultants from a breadth of specialties, clinic staff and estates. Each trainee (10 in total) received a rota at the start of each placement which included seven specific clinics for them to attend. It was made clear that these sessions were compulsory and that an exception report would be filed in the event of nonattendance. A form was provided to show evidence at clinic and also provide feedback. The MUM was responsible for rearranging clinics should leave be taken on a proposed clinic day.

\section{Results and discussion}

Ten CMTs were allocated to 7 clinic slots over a 4-month period. Of the 70 clinics scheduled there is documented evidence of attendance for $53 \%$ of scheduled clinics. However, qualitative feedback indicates that $80 \%$ of trainees were on target to meet their minimum requirements suggesting that there may be clinic attendance lacking documentation. $80 \%$ of trainees had also attended clinics in addition to their scheduled allocations. All of the CMTs completed at least one work-based placed assessment during their clinic time. All received informal feedback. No trainees completed a reflection on their clinic attendance. No exception reports were filed despite there being non-attendance at clinics.

This is the first cycle of this educational quality improvement project and we hope during the next cycle to improve clinic attendance among trainees with greater emphasis on reporting non-attendance and allowing time for trainees to feedback on their experience. We must explore the reasons for non-attendance and encourage reporting of this so that the reasons can be explored and if possible solutions sought. The trust now has a rolling rota with input from the MUM to ensure there are clinic slots available for trainees and we hope that this will improve the overall trainee experience for CMTs.

\section{Conclusion}

Implementation of a MUM at Warrington Hospital has allowed for a dedicated rota to be designed to enable CMT clinic attendance while ensuring patient safety on the wards is not negatively impacted. Attendance is presumed to have improved although there was no baseline data to back up this assumption. Further work is required to make certain trainees document their attendance at clinic and that clear reasons are documented for non-attendance. 